\title{
Real-time assessment of cigarette smoke particle deposition in vitro
}

\author{
Jason Adamson, Sophie Hughes, David Azzopardi, John McAughey and Marianna D Gaça*
}

\begin{abstract}
Background: Recently there has been a rapid increase in approaches to assess the effects of cigarette smoke in vitro. Despite a range of gravimetric and chemical methods, there is a requirement to identify simpler and more reliable methods to quantify in vitro whole smoke dose, to support extrapolation and comparisons to human/in vivo dose. We have previously characterised an in vitro exposure system using a Borgwaldt RM20S smoking machine and a chamber exposing cellular cultures to whole smoke at the air-liquid interface. In this study we demonstrate the utility of a quartz crystal microbalance (QCM), using this exposure system, to assess real-time cigarette smoke particulate deposition during a 30 minute smoke exposure. Smoke was generated at various dilutions (1:5-1:400, smoke:air) using two cigarette products, 3R4F Kentucky reference and 1 mg commercially available cigarettes. The QCM, integrated into the chamber, assessed particulate deposition and data generated were compared to traditional chemical spectrofluorometric analysis.

Results: The QCM chamber was able to detect mass differences between the different products within the nanogram range. 3R4F reference cigarette smoke deposition ranged from $25.75 \pm 2.30 \mu \mathrm{g} / \mathrm{cm}^{2}$ (1:5) to 0.22 $\pm 0.03 \mu \mathrm{g} / \mathrm{cm}^{2}$ (1:400). $1 \mathrm{mg}$ cigarette smoke deposition was less and ranged from $1.42 \pm 0.26 \mu \mathrm{g} / \mathrm{cm}^{2}$ (1:5), to 0.13 $\pm 0.02 \mu \mathrm{g} / \mathrm{cm}^{2}$ (1:100). Spectrofluorometric analysis demonstrated statistically significant correlation of particulate deposition with the QCM $(p<0.05)$, and regression $R^{2}$ value were $97.4 \%$. The fitted equation for the linear model which describes the relationship is: $\mathrm{QCM}=-0.6796+0.9744$ chemical spectrofluorescence.
\end{abstract}

Conclusions: We suggest the QCM is a reliable, effective and simple tool that can be used to quantify smoke particulate deposition in real-time, in vitro and can be used to quantify other aerosols delivered to our chamber for assessment.

Keywords: QCM, Cigarette smoke, In vitro exposure chamber, Dosimetry

\section{Background}

Cigarette smoke is a complex and dynamic aerosol consisting of at least 5,600 chemicals and toxicants found across two phases, the particulate (tar) and vapour phase [1]. Recently, there has been a rapid increase in the development of systems for in vitro biological and toxicological assessment of whole smoke [2-11]. However, despite these advancements there have not been consistent approaches in reporting accurately the dose of whole smoke delivered to in vitro cultures.

Understanding dosimetry is essential when attempting to mimic or extrapolate human smoking behavior and in vivo doses to in vitro models. Whole smoke dose is

\footnotetext{
* Correspondence: Marianna_Gaca@bat.com

British American Tobacco, Group R\&D, Regents Park Road, Southampton, SO 15 8TL, UK
}

\section{(Chemistry Central}

dependent on the machine used to generate, dilute and deliver smoke and is variously described as a percentage of smoke, a fraction of smoke, ratios of smoke to air, puff number, total exposure of micrograms per insert, or as a flow rate of mixing air and vacuum applied to a smoke dilutor $[2,3,5,6,9-11]$. This is a relatively new and challenging field but is an increasingly important point of discussion within the industry. On a broader note, the need to quantify absolute chemical or particle deposition in in vitro model systems is of increasing importance to scientists and regulators for consistent interpretation of disease model end-points versus a defined biologically effective dose [12,13].

There are a number of reported studies quantifying components of either the particulate or vapour phase as a means of assessing dose. Solanesol is the most 
common constituent measured in the particulate phase [14], and carbon monoxide in the gas phase [6]. Most dosimetry measurements of cigarette smoke are of the particulate phase due to the challenges of measuring individual components in the vapour phase, especially at higher smoke dilutions. However, many of the methodologies involved are complex, often off-line and involve many steps where errors or loss of precision could be introduced, and there is no general consensus on the most appropriate approach. There is therefore a requirement for a simple, more reliable and a standard method to be used for whole smoke in vitro dose assessments.

The quartz crystal microbalance (QCM) is a sensitive gravimetric balance capable of measuring and detecting changes in mass, within the nanogram range, of thin adherent films [15-17], and has been used as such since the 1950's following pioneering scientific work by Sauerbrey [18]. It makes use of the piezoelectric effect associated with all quartz crystals. Mechanical and electrical stress applied to the crystal, when incorporated into an electrical circuit, produces an electric potential, and when applied to the crystal produces mechanical deformation on the crystal $[16,19]$. These properties, when employed, generate waves whose frequencies are influenced by changes in mass at the crystal surface [20].

The QCM consists of a thin quartz disc held between two electrodes, often made of gold, combined with software technology capable of monitoring and recording changes in frequency. The rate of oscillation of the quartz crystal is directly related to its thickness (when other variables such as temperature and humidity remain constant), therefore crystals of the same specific thickness will oscillate at the same resonant frequency $[19,21]$. As mass is added onto an oscillating quartz crystal, its effective thickness is increased. This change in thickness correlates directly to a change in oscillation frequency: the greater the deposition of a given substance onto the crystal surface, the lower the frequency of oscillation [21]. Sauerbrey's equation [18] can be employed to convert the frequency shift into the mass per unit area of thin film deposition [16,19]. Under ideal conditions, it is assumed that the deposited mass forms a monolayer, hence changing the effective thickness of the crystal as described with the deposited mass fully coupled to the crystal. In practice, the smoke particles are approximately $300 \mathrm{~nm}$ count median diameter (cmd), and while not initially forming a monolayer, they are sufficiently small that they would not be expected to oscillate independently of the crystal.

QCMs have been used for a wide variety of applications, one of the most common being the monitoring of water pollution [17]. Many advances have also been made in biological disciplines where QCMs have been used to detect entities as small as virus nanoparticles
[22] and peptide membrane binding dynamics [23]. QCMs have also been utilised to quantify different types of smoke by mass, such as outdoor tobacco smoke [24] and blood and bone associated aerosols/cautery smoke from orthopaedic surgery [25], as well as ultrafine particles in vitro [15]; however, to our knowledge a QCM has not yet been reported to quantify cigarette smoke dosimetry in vitro within an exposure chamber.

In this study we present a novel application of a QCM, to assess the real-time deposition of cigarette smoke in vitro. We have previously published studies outlining the design of an exposure chamber used to expose in vitro cultures at the air-liquid interface (ALI) to whole smoke (Figure 1), [2,9]. Furthermore, we have demonstrated repeatable and accurate whole smoke dilution (ranging from 1:2-1:4,000 smoke:air, volume:volume) and delivery of cigarette smoke using a commercially available Borgwaldt RM20S smoking machine (Borgwaldt-kc, Hamburg, Germany) [2,6] and successful applications in vitro $[7,9,11]$. In this study we have investigated the ability of a QCM, integrated into a whole smoke chamber (Figure 2), to detect mass differences between two different cigarette types of different tar deliveries, a 3R4F Kentucky reference cigarette (pack tar value of $9.4 \mathrm{mg} /$ cigarette), and a $1 \mathrm{mg}$ pack tar commercially available product. Cigarettes were smoked at various dilutions on a Borgwaldt RM20S smoking machine and real-time results on the QCM were used to quantify absolute deposition within the chamber during a 30 minute whole smoke exposure, consistent with existing in vitro exposure durations within our laboratories. To compare QCM real-time deposition data, a traditional chemical spectrofluorometric method to quantify particulate deposition, previously described [2], was used.

Results from this study demonstrated the QCM was able to discriminate mass balance from two different products accurately, even on a puff-by-puff basis, within the scope of existing methodology. The integrated QCM delivered easily and reliably, real-time whole smoke mass measurements, at nanogram levels within manufacturer's specification (at a resolution of $10 \mathrm{ng} / \mathrm{cm}^{2} / \mathrm{s}$ with a lower detection limit of $20 \mathrm{ng} / \mathrm{cm}^{2} / \mathrm{h}$ (http://www.vitrocell. com- product info download)), and demonstrated quantitative measurements and an achievable dose response. This device shows potential to be used to quantify other aerosols delivered to our chamber for in vitro assessment and as a possible tool for other in vitro exposure systems.

\section{Results}

\section{QCM whole smoke quantification}

The QCM, integrated within the exposure chamber, was able to measure whole smoke particulate deposition on 
A

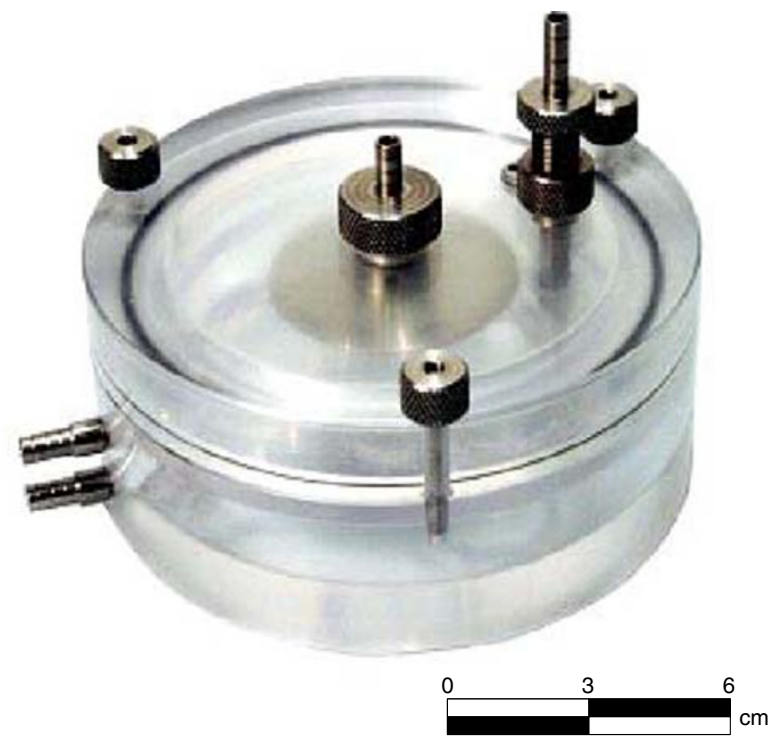

B

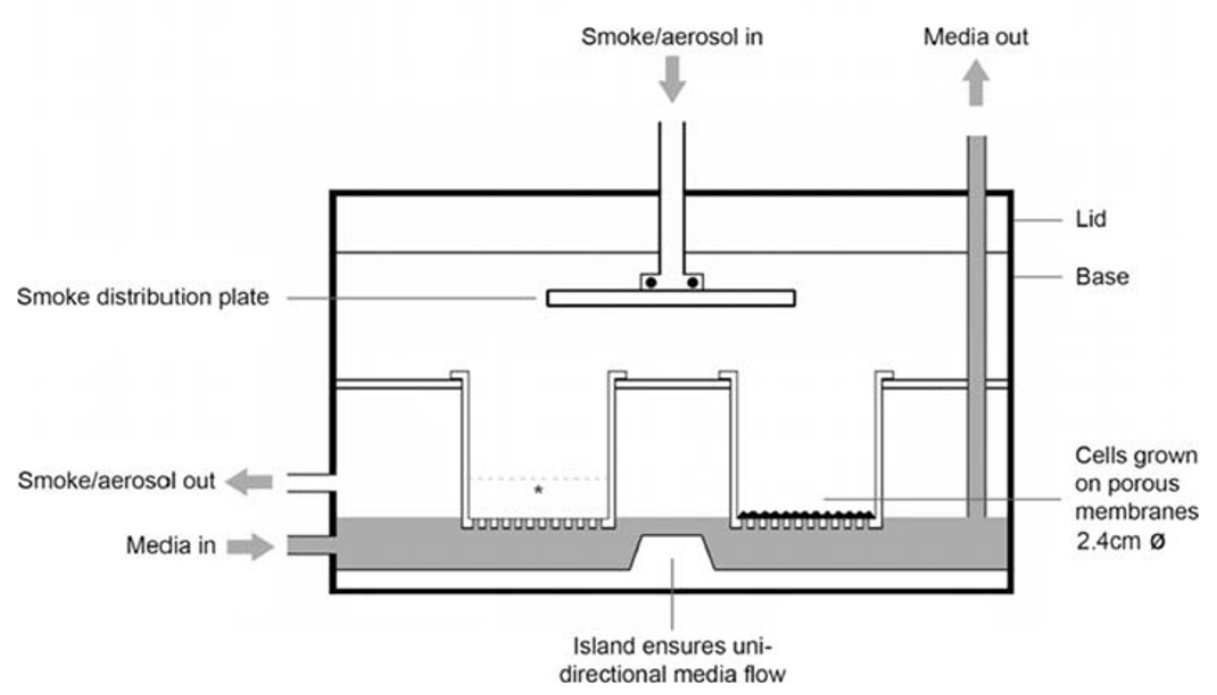

Figure 1 British American Tobacco's standard exposure chamber used for in vitro exposures to whole smoke at the ALI [A], and a schematic cross-section [B]. For extraction of deposited particulate matter for spectrofluorescence analysis, * illustrates the level reached by $2 \mathrm{ml}$ extraction solvent when added to the exposed cell culture insert, rising $0.56 \mathrm{~cm}$ up the inner wall (diagram adapted from [11]).

cell culture inserts within the nanogram range. Smoke was generated at a variety of dilutions from two different cigarette products. During a 30 minute whole smoke exposure, the integrated QCM could detect particulate deposition on a puff-by-puff basis with both the 3R4F reference cigarette (Figure 3A) and also the $1 \mathrm{mg}$ cigarette (Figure 3B). Stable (flat) profiles were observed both before and after smoke generation. There then appeared to be a steady mass gain from first puff, followed by a repeated pattern of response per cigarette puff: a rapid initial increase in mass then a slower but more sustained increase in mass, concluding in a slight decease in mass (outlined in figure 3A). This 'unit' of the repeated pattern represents one puff from the machine entering the chamber and is particularly evident with the higher tar delivery 3R4F reference cigarette, with 30 distinct repeating units per puff (Figure $3 \mathrm{~A}$ ). The mass increased with deposition of particles as smoke filled the chamber over 8 seconds, which would represent high initial deposition by turbulent mixing during filling. When the syringe smoke line valve closes after the puff has exhausted, smoke then sits in the chamber under still conditions for 52 seconds, during which a consistent slight increase in mass is observed, probably through a 
A
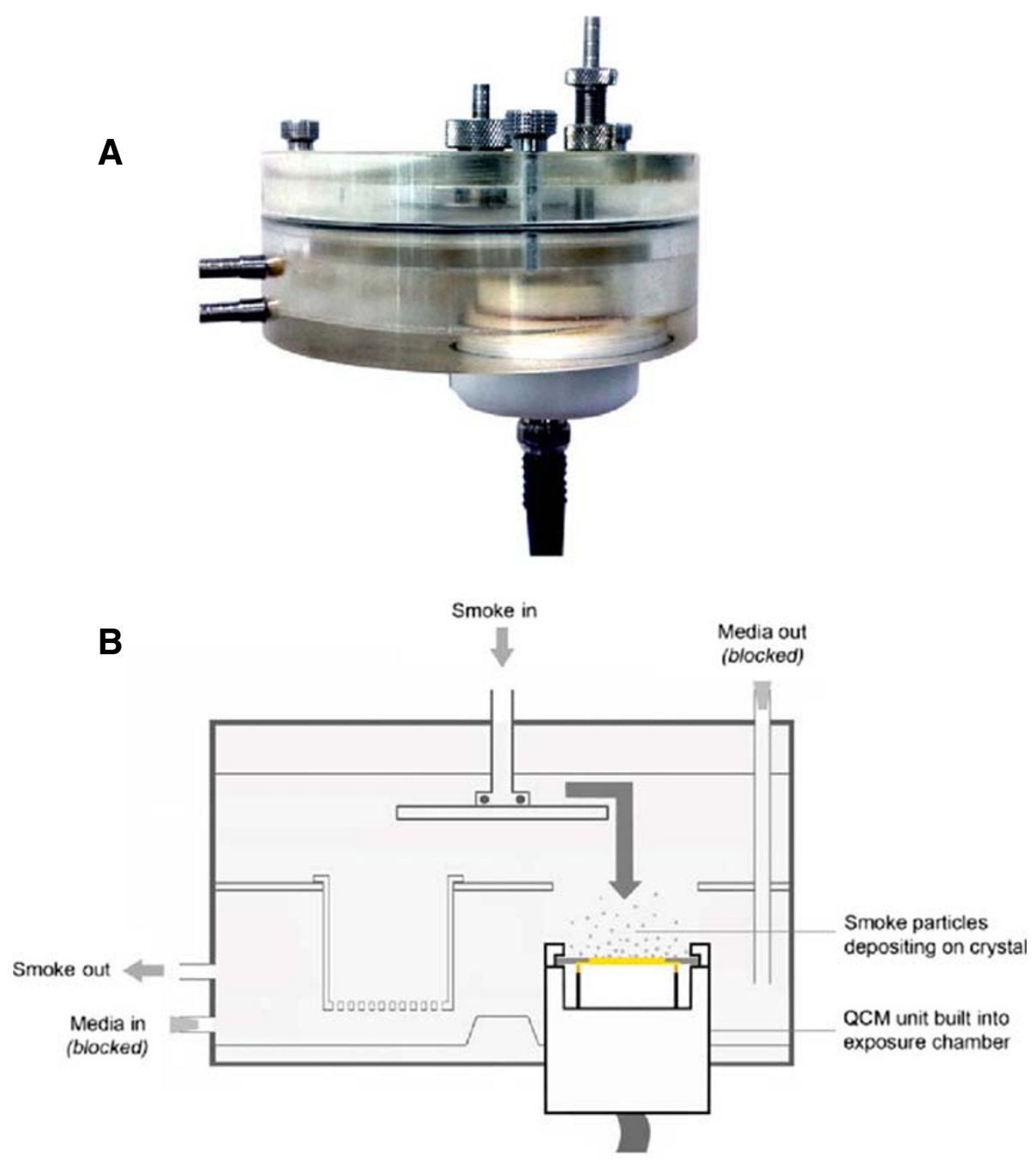

C

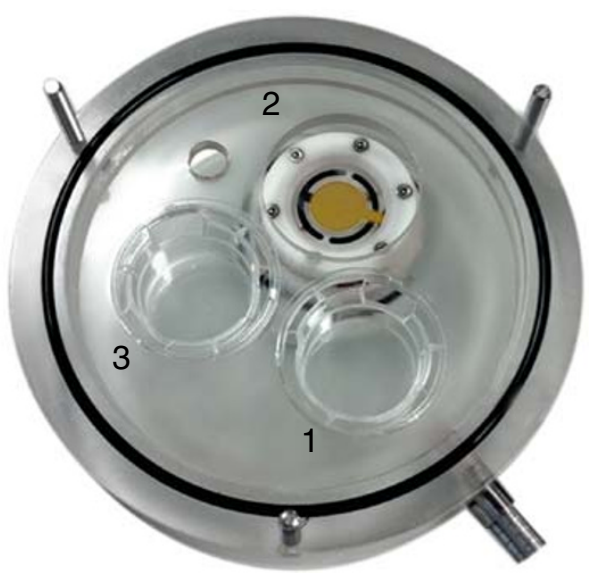

Figure 2 A single QCM unit housed within the exposure chamber (side view) [A]; a schematic cross-section of the QCM exposure chamber $[B]$; and a top view of the chamber base showing that the QCM fits into and replaces the position of one of the three cell support inserts [C2], allowing the remaining two positions to house cell support inserts if required for parallel exposure [C1, 3]. Internal surface area and volume changes upon installation of the single QCM unit were nominal, compared to the original chamber geometry. Crystal $\varnothing=2.5 \mathrm{~cm}$; cell support insert $\varnothing=2.4 \mathrm{~cm}$; crystal's gold electrode $\varnothing=1.3 \mathrm{~cm}$. 
A

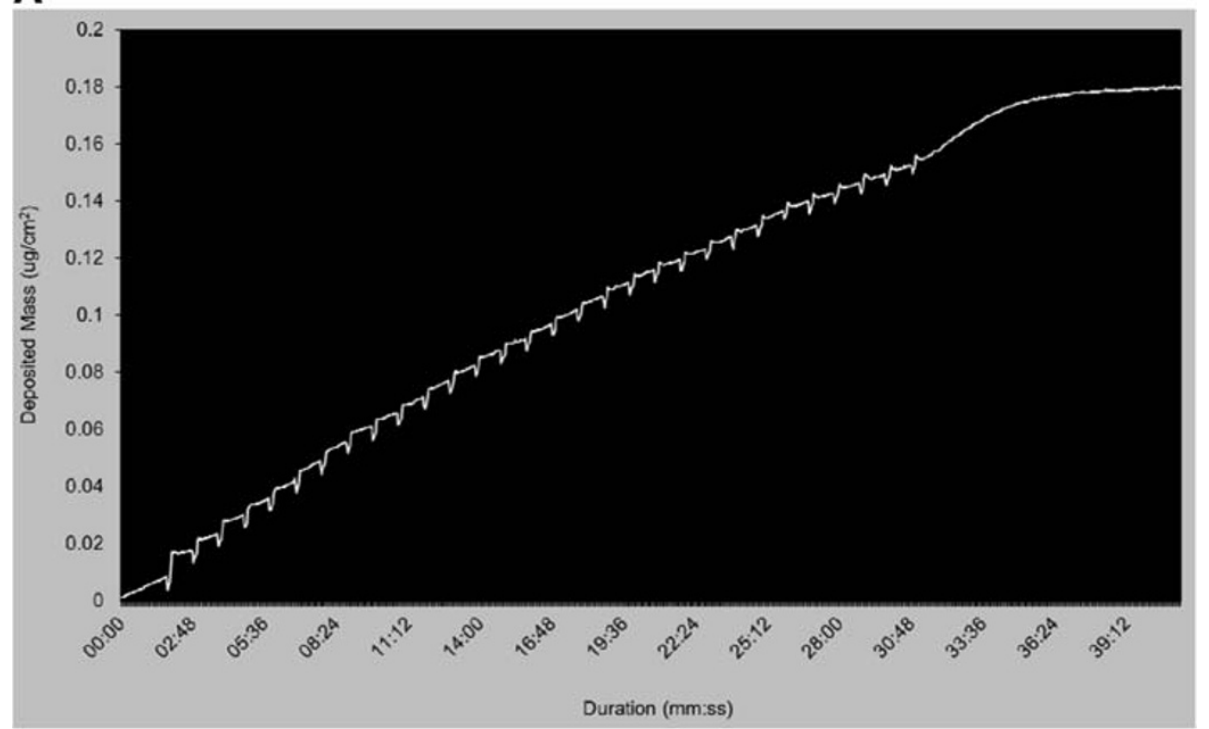

B

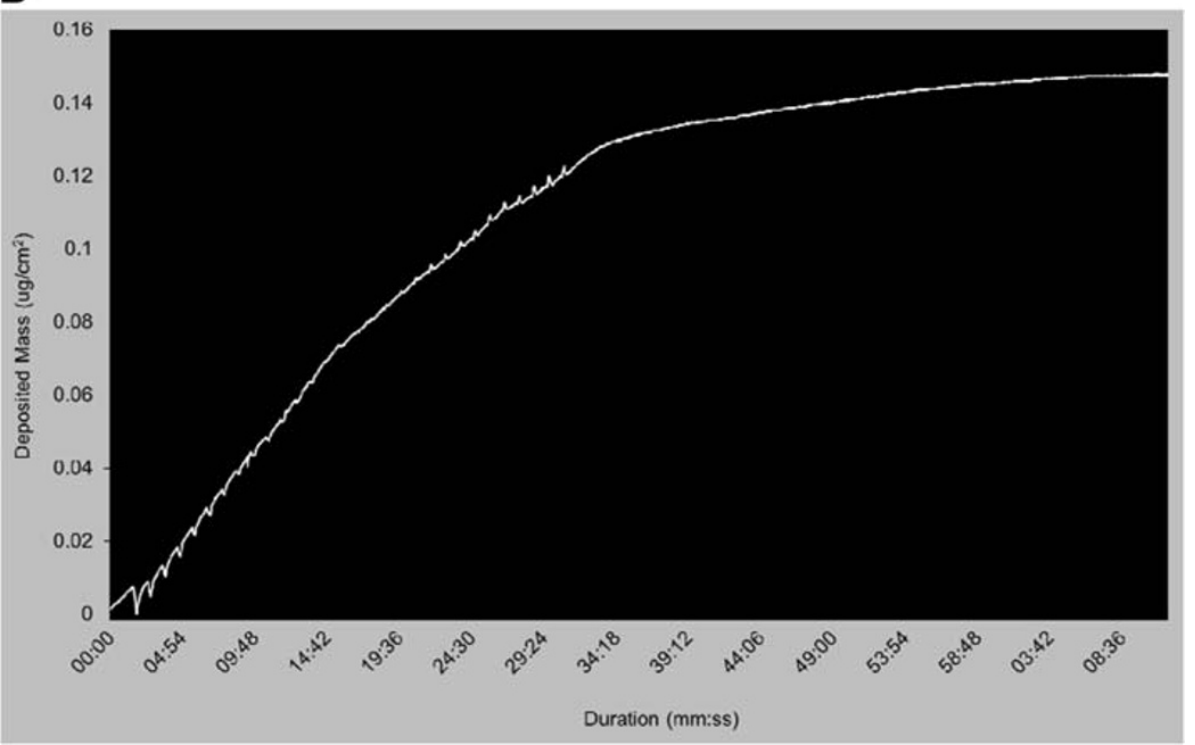

Figure 3 Real-time traces of QCM deposited mass in the exposure chamber, showing a 3R4F cigarette smoked at a dilution of 1:400 $[\mathrm{A}]$, and a $1 \mathrm{mg}$ commercially available cigarette smoked at a dilution of 1:100 [B]. Observe the plateau phase after the smoke run has finished, showing that stabilisation of the crystal is constant after approximately 10 minutes.

combination of sedimentation and diffusion. Finally, as the smoke is exhausted from the syringe and the next diluted puff enters the chamber over 8 seconds, a slight decrease in mass is observed, probably due to evaporation of some smoke vapour phase components from the surface of the crystal caused by the increase in airflow, momentarily reducing the mass. The trend is repeated per puff and the overall trend of mass increases reproducibly during exposure.

Both cigarette types produced an expected dose response. More particulate material was deposited at lower dilutions (higher concentrations) of smoke compared to less particulate being deposited at higher dilutions (lower concentrations) of smoke. With the 3R4F reference cigarette, 5 dilutions were studied ranging from 1:51:400 (smoke:air, volume:volume) (Figure 4A). Deposited mass detected within the chamber ranged from 25.75 $\pm 2.30 \mu \mathrm{g} / \mathrm{cm}^{2}\left(25,750 \mathrm{ng} / \mathrm{cm}^{2}\right)$ at the lowest dilution of smoke (1:5), to $0.22 \pm 0.03 \mu \mathrm{g} / \mathrm{cm}^{2}\left(220 \mathrm{ng} / \mathrm{cm}^{2}\right)$ at the highest dilution of smoke (1:400) (Table 1).

For the $1 \mathrm{mg}$ commercial cigarette, 4 dilutions were studied ranging from 1:5-1:100 (Figure 4B). The highest 

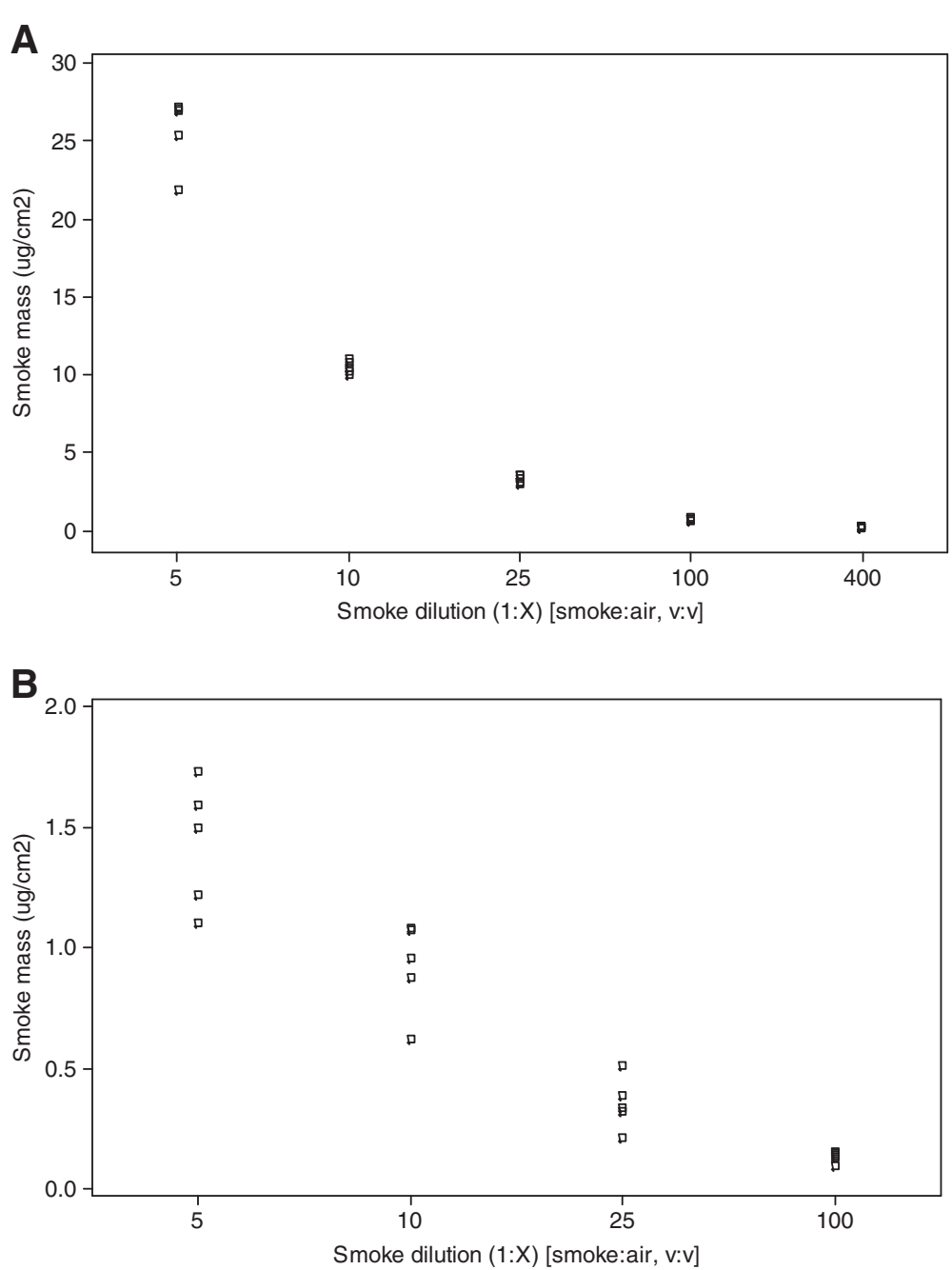

Figure 4 Individual value plots showing QCM particulate deposition over a range of whole smoke dilutions tested for 3R4F reference cigarettes $[A]$ and $1 \mathrm{mg}$ commercially available cigarettes $[B]$, for a 30 minute whole smoke exposure $(n=5)$.

dilution of smoke tested with the 3R4F reference cigarette (1:400) was not used for the $1 \mathrm{mg}$ product as, although not lower than the absolute detection limit, it was low enough for us not to have confidence in the data obtained, based on the natural drift of the crystal, but more so due to the time taken to stabilise it prior to each run. To demonstrate, the QCM detects at a resolution of $10 \mathrm{ng} / \mathrm{cm}^{2} / \mathrm{s}$; at the lowest dilution of 1:100 for the $1 \mathrm{mg}$ cigarette the value obtained was $0.13 \mu \mathrm{g} / \mathrm{cm}^{2} / 60 \mathrm{~s}$ which would notionally equate to $20 \mathrm{ng} / \mathrm{cm}^{2} / \mathrm{s}$, close to the level of resolution. Overall, deposited mass detected within the chamber was notably less than for the $3 \mathrm{R} 4 \mathrm{~F}$ cigarette and ranged from $1.42 \pm 0.26 \mu \mathrm{g} / \mathrm{cm}^{2}\left(1,420 \mathrm{ng} / \mathrm{cm}^{2}\right)$ at the lowest dilution of smoke (1:5), to $0.13 \pm 0.02 \mu \mathrm{g} / \mathrm{cm}^{2}$ $\left(130 \mathrm{ng} / \mathrm{cm}^{2}\right)$ at the highest dilution of smoke $(1: 100)$ during the 30 minutes smoke exposure (Table 1).

Table 1 Particulate deposition data for 3R4F reference and $1 \mathrm{mg}$ commercially available cigarettes over a range of smoke dilutions tested during a $\mathbf{3 0}$ minute exposure

\begin{tabular}{|c|c|c|c|c|c|c|c|c|c|c|}
\hline \multirow{3}{*}{$\frac{\text { Dilution (1:X) }}{\text { Method }}$} & \multicolumn{10}{|c|}{ mean deposited mass $\left(\mu \mathrm{g} / \mathrm{cm}^{2}\right) \pm S D$} \\
\hline & \multicolumn{2}{|c|}{5} & \multicolumn{2}{|c|}{10} & \multicolumn{2}{|c|}{25} & \multicolumn{2}{|c|}{100} & \multicolumn{2}{|c|}{400} \\
\hline & QCM & Fluor & QCM & Fluor & QCM & Fluor & QCM & Fluor & QCM & Fluor \\
\hline 3R4F cigarette & $25.75 \pm 2.30$ & $26.94 \pm 5.57$ & $10.51 \pm 0.42$ & $12.69 \pm 3.49$ & $3.28 \pm 0.24$ & $4.54 \pm 1.60$ & $0.68 \pm 0.09$ & $1.60 \pm 0.27$ & $0.22 \pm 0.03$ & $0.57 \pm 0.20$ \\
\hline $1 \mathrm{mg}$ cigarette & $1.42 \pm 0.26$ & $1.36 \pm 0.48$ & $0.92 \pm 0.19$ & $0.81 \neq$ & $0.35 \pm 0.11$ & $0.45 \neq$ & $0.13 \pm 0.02$ & $0.28 \neq$ & - & - \\
\hline
\end{tabular}

\# Interpolated values, taken from a standard curve of data generated within the same range but at different dilutions of 1:20, 1:50, 1:200 (smoke:air, v; v). 
Standard deviation was noticeably highest at the lowest dilution of smoke (1:5) for both products tested. This may be due to the complex and concentrated nature of cigarette smoke at this dilution, the puff-by-puff measurement and cigarette by cigarette variability which is commonly observed. After every smoke run a plateau phase was observed where mass neither increased or decreased significantly, and usually took an additional 10 minutes after the smoke run. The stability at this end point demonstrated no more volatile loss and therefore robustness of the tool.

\section{Comparison of QCM using chemical spectrofluorometric analysis}

To compare the utility of the QCM to routine methods, independent deposition fluorescence analyses were conducted in separate exposure chambers. Particulate matter depositing during the whole smoke exposures was eluted from the inserts and quantified using fluorescence spectroscopy. The particulate deposition data were plotted against the QCM data (in the same dilution range for each product tested) as a regression fitted line plot for both cigarette types (Figure 5).

The linear range of the results from the spectrofluorescence method was over 2 orders of magnitude for smoke dilution (1:5-1:400 for the 3R4F reference cigarette and 1:5-1:200 for the $1 \mathrm{mg}$ commercial cigarette) with particle mass deliveries of $26.94-0.57 \mu \mathrm{g} / \mathrm{cm}^{2}$ and $1.36-0.28 \mu \mathrm{g} / \mathrm{cm}^{2}$ respectively (Table 1 ). As with the QCM results, the chemistry data showed that there was a positive correlation between smoke concentration and particulate depositing on the insert for both products smoked. The fitted line plot, $\mathrm{R}^{2}$ value was $97.4 \%$ (Figure 5 ) of whole smoke particulate deposition over a range of smoke dilutions for both the 3R4F reference cigarette and a $1 \mathrm{mg}$ commercially available cigarette. For both cigarette types the relationship between the two methods was statistically significant $(\mathrm{p}<0.05)$."

\section{Discussion}

We have presented a study demonstrating a novel and simple system to quantify in vitro cigarette smoke deposition in real-time. Although used for numerous mass measurement applications in fields such as pollution, biological, environmental and occupational monitoring $[15,17,24,25]$, to our knowledge there is no published information on the use of the QCM for in vitro whole cigarette smoke assessment. A QCM was integrated into our established in vitro exposure chamber to quantify real-time deposition of cigarette smoke particles onto cell cultures. Furthermore, with the current switch from liquid to ALI exposures of aerosols in vitro [13], and increasing physiological relevance, this ALI method of particle quantification is even more important.

The QCM chamber enabled the quantification of a range of mass values per surface area for a given dilution of smoke with air, for example $25.75 \pm 2.30 \mu \mathrm{g} / \mathrm{cm}^{2}$ to 0.22 $\pm 0.03 \mu \mathrm{g} / \mathrm{cm}^{2}$ mass range for 3R4F reference cigarettes dilution range 1:5-1:400 (v:v), and $1.42 \pm 0.26 \mu \mathrm{g} / \mathrm{cm}^{2}$ to $0.13 \pm 0.02 \mu \mathrm{g} / \mathrm{cm}^{2}$ mass range for $1 \mathrm{mg}$ commercial cigarettes dilution range 1:5-1:100 (v:v) (Table 1). A clear dose response was demonstrated and detected by the QCM at

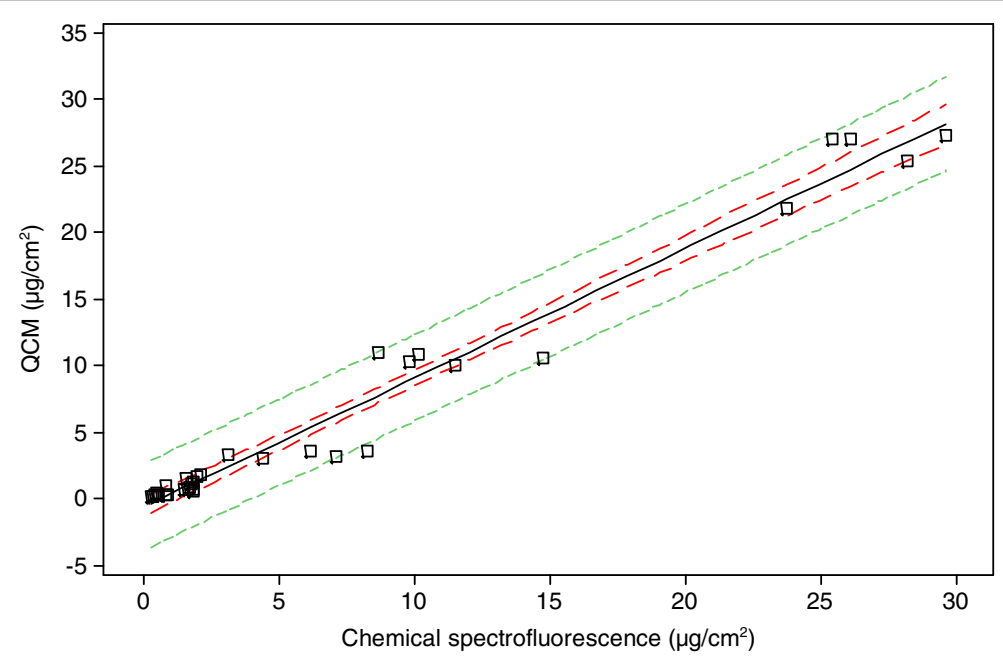

Figure 5 Fitted line plot comparison of QCM and chemical spectrofluorescence assessment of whole smoke particulate deposition over a range of smoke dilutions for both $3 R 4 F$ reference cigarette and a $1 \mathrm{mg}$ commercially available cigarette $\left(R^{2}=97.4 \%\right)$. Solid black line = Regression, dashed red line $=95 \% \mathrm{Cl}$ (confidence intervals), dotted green line $=95 \% \mathrm{PI}$ (prediction intervals). For both cigarette types the relationship between the two methods was statistically significant $(p<0.05)$; this was based on a sample size large enough $(n=105)$ to obtain a precise estimate of the strength of the relationship. The fitted equation for the linear model which describes the relationship is: $\mathrm{QCM}=-0.6796+0.9744$ chemical spectrofluorescence. 
various dilutions of cigarette smoke from two different tar delivery products (Figure 4). The QCM was sensitive enough to detect low levels of deposited matter at a resolution of $10 \mathrm{ng} / \mathrm{cm}^{2} / \mathrm{s}$ with a lower detection limit of $20 \mathrm{ng} / \mathrm{cm}^{2} / \mathrm{h}$ (http://www.vitrocell.com product info download) allowing the assessment of low $1 \mathrm{mg}$ tar delivery products at high dilutions with air (Figure $3 \mathrm{~B}$ ).

The QCM data obtained in real-time were compared with a traditional chemical fluorescence method of particulate quantification; the overall distribution demonstrated a good correlation between the two described methods of particle detection, and regression analysis demonstrated that the relationship between the two methods was significant. The observed difference between the QCM and fluorescence measurements (Figure 5) could be accounted by the fluorescence method solvent extraction process, which involves multiple steps off-line and a possible effect of surface area correction to account for the total surface area of the well washed rather than just the base area of the well. It is also possible that there may be an effect of evaporation of vapour phase components from the surface of the QCM, or semi-volatiles redistributing due to a dynamic equilibrium between the particulate and vapour phase.

Deposition in the well will occur during the eight second filling period for the chamber, where turbulent mixing will dominate deposition to all surfaces and this is characterised by a sharp increase of deposited mass in the real-time QCM trace (Figure 3A). This is followed by a 52 second still period where sedimentation (to the well base) and diffusion (to all surfaces) will predominate. Calculated values [26] for a $390 \mathrm{~nm}$ volume median diameter smoke droplet entering the chamber at $310 \mathrm{~K}$ [2] represent a settling velocity of $6.28 \mathrm{E}-06 \mathrm{~m} / \mathrm{s}$ with a mean displacement of $0.33 \mathrm{~mm}$ over $52 \mathrm{~s}$. Over the same $52 \mathrm{~s}$ the $\mathrm{rms}$ displacement by diffusion is approximately $0.10 \mathrm{~mm}$. These relatively small displacements are consistent with the slower rise in mass observed during the still phase. Thus we have chosen to surface area correct the spectrophotometric data for the full washed area of the well to represent the rapid deposition during mixing.

For the apparent transient mass loss at the end of the smoke residence period, two mechanisms for mass under-reporting or loss from the QCM have been described previously. The first is generally observed for the Tapered Element Oscillating Microbalance (TEOM), which shares the QCM measurement principle, albeit in a different geometry. In part, combustion particles such as diesel soot may form chain-like aggregates where only part of the chain attaches to the microbalance, hence the particle mass is not fully coupled to the crystal mass [27]. Tobacco smoke is a liquid spherical droplet [28] and as such its mass is expected to fully couple to the microbalance. As noted earlier, under ideal conditions, it is assumed that the deposited mass forms a monolayer, fully coupled to the crystal. In practice, the smoke particles are approximately $300 \mathrm{~nm}$ count median diameter (390 nm volume median diameter) [2], and while not initially forming a monolayer, they are sufficiently small that they would not be expected to oscillate independently of the crystal.

It has also been observed that mass loss may occur with evaporation of semi-volatile components of the aerosol mass, particularly where the balance is heated for water elimination, for example in ambient air sampling [29] and diesel sampling above. For tobacco smoke, nicotine and water, accounting for approximately $14 \%$ of the aerosol mass for the 3R4F cigarette are effectively semivolatile in this measurement context [30]. It has also been reported that numerous chemical species in tobacco smoke have been demonstrated to be semi-volatile in in vitro exposure systems [31].

Direct output of the microbalance shows puff-by-puff increases in absolute mass and a general increase in deposited mass during the inter-puff period where smoke is held in the exposure chamber. However, anomalies are observed with small transient mass losses particularly when the chamber is filling and emptying. Future work using a range of tar delivery cigarettes will help further understand the sensitivity of the QCM, but also to resolve gradient differences between products we have observed, and to discriminate deposition to the walls and base of the well.

\section{Materials and methods}

\section{Whole cigarette smoke generation}

Whole cigarette smoke was generated for QCM and chemical fluorescence assessment using a Borgwaldt RM20S smoking engine (Borgwaldt-kc, Hamburg, Germany) as previously described [2]. Smoke dilutions were programmed as a ratio of smoke to air; for QCM experiments 5 dilutions were chosen in the range 1:5-1:400 (smoke:air, volume:volume) ( $\mathrm{n}=5$ per dilution) and for deposition experiments 7 dilutions were chosen in the range 1:5-1:400 $(n=4-6)$. For all experiments, the machine smoked for 30 minute duration at the ISO smoking regime (35 $\mathrm{ml}$ puff over 2 seconds, once a minute [32]). However, rather than smoking to standard butt length, duration was controlled by puff number; 5 cigarettes were smoked at 6 puffs each (30 minutes total). During exposure, smoke filled the chamber over 8 seconds and was then left (for 52 seconds) until the next puff of diluted smoke was delivered to the chamber - this is batch mode smoking, as opposed to continuous flow delivery of smoke. After machine smoking for the QCM, the device was left for an additional 10 minutes for residual smoke to settle in the chamber and the real-time deposition values to plateau. Two types of cigarettes were 
tested, 9.4 mg pack tar 3R4F reference cigarettes (University of Kentucky, Kentucky, USA) and $1 \mathrm{mg}$ pack tar commercially available cigarettes. Pack tar refers to the value of the total particulate matter (TPM) of the cigarette smoke trapped on a Cambridge filter pad less the value of nicotine and water content; therefore pack tar is also termed 'nicotine free dry particulate matter' or NFDPM. Table 2 shows the characteristics of these products. Cigarettes were conditioned for a minimum of 48 hours before smoking ( $60 \pm 3 \%$ relative humidity, 22 $\pm 1{ }^{\circ} \mathrm{C}$ ) according to ISO 3402:1999 [33].

\section{The QCM exposure chamber module}

The previously described BAT exposure chamber $[2,9]$ (Figure 1), was installed with a commercially available QCM unit (5 MHz AT cut quartz crystals held between two $\mathrm{Au} / \mathrm{Cr}$ polished electrodes, 1 inch $(2.5 \mathrm{~cm})$ diameter as described by Mülhopt et al., 2009 [15], with associated software which converted oscillator frequency into mass per surface area $\left(\mathrm{ng} / \mathrm{cm}^{2}\right)$ (Vitrocell ${ }^{\circledR}$ Systems GmbH, Waldkirch, Germany), (Figure 2). The QCM read at a resolution of $10 \mathrm{ng} / \mathrm{cm}^{2} /$ second, as per the manufacturer's specification (http://www.vitrocell.com product info download). The active area of the crystal which records deposited mass is the gold electrode in the center (Figure 2C) measuring $13 \mathrm{~mm}$ diameter, however the software provided converts the mass detected on this electrode to $\mathrm{ng} / \mathrm{cm}^{2}$.

Before exposing to whole cigarette smoke, the QCM chamber was acclimatised to ensure quartz crystal stability for a minimum of 10 minutes at $37^{\circ} \mathrm{C}$, with the baseline re-set to zero at 1 minute increments. During the whole cigarette smoke generation and exposure phase, the QCM took mass readings every 2 seconds during the

Table 2 Physical and chemical characteristics of the cigarettes tested in this study

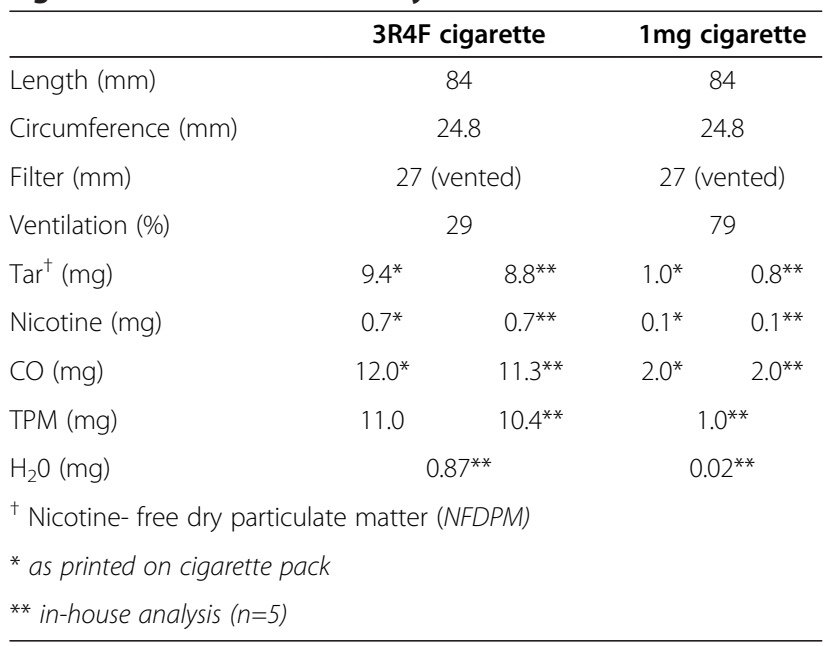

3R4F cigarette tar/TPM values obtained from the University of Kentucky website (http://www.ca.uky.edu/refcig).
30 minute exposure and reported as mass per unit area. Cell culture media and/or cells were not included in the chamber for these mass measurements (although capable) hence media-in and media-out ports were blocked (Figure 2B). After whole smoke exposure, quartz crystals were cleaned using $70 \%$ ethanol and wiping with a soft lint-free tissue.

\section{Deposition quantification using chemical fluorescence analysis}

To compare QCM generated deposition data, chemical spectrofluorescence analysis was used to quantify particulate deposition within the exposure chamber during smoke exposure at a range of dilutions generated from either 3R4F reference cigarettes or $1 \mathrm{mg}$ commercially available cigarettes, as described previously [2]. These experiments were conducted independently from the QCM measurements, in separate exposure chambers and at a different time, but over the same exposure range and at exactly the same experimental conditions for whole smoke generation.

Briefly, after smoke exposure, deposited particulate material was extracted from inserts (6 well plate format) using $2 \mathrm{ml}$ high performance liquid chromatography (HPLC) grade methanol (Hayman Ltd, Essex, UK) and agitation on a plate shaker at $150 \mathrm{rpm}$ for 10 minutes. Extracts were analysed by HPLC using an Agilent 1100 Series (Agilent, UK). Fluorescence was detected with an Agilent standard FLD cell (Agilent, UK) at excitation and emission wavelengths of $286 \mathrm{~nm}$ and $350 \mathrm{~nm}$ respectively. Extract particulate (gravimetric particulate matter) concentrations were calculated using standard calibration curves and the blank insert results subsequently subtracted from the extract values. Data were converted to particulate deposition in mass per surface area.

Sample extracts were quantified against an external standard prepared from filter extracted particulate matter (TPM) for the 3R4F reference or $1 \mathrm{mg}$ cigarettes, as described previously [2,9]. The standard calibration curve was prepared from PM concentrations ranging from 0.48 - $38 \mu \mathrm{g} / \mathrm{ml}$. To compare the QCM with the spectrofluorometric data graphically, the same range of whole smoke dilutions were plotted against each other for both methods: 1:5-1:400 for the 3R4F cigarette and 1:5-1:100 for the $1 \mathrm{mg}$ product.

The insert membrane diameter was $2.4 \mathrm{~cm}$ (6 well plate format) but with the addition of $2 \mathrm{ml}$ of methanol for elution, an extra $0.56 \mathrm{~cm}(+\leq 0.2 \mathrm{~cm}$ during agitation on the plate shaker, as measured during plate agitation) of insert wall was washed (Figure 1*). This increased the potential total eluted surface area from $4.52 \mathrm{~cm}^{2}$ (insert membrane only) to a maximum of $10.24 \mathrm{~cm}^{2}$ assuming a maximum wash height of $0.76 \mathrm{~cm}$. For comparative purposes, the spectrophotometric data were converted 
to mass per $\mathrm{cm}^{2}$ using the $10.24 \mathrm{~cm}^{2}$ area value and this was addressed further in the discussion section of this paper.

\section{Statistics}

Data were reported as a mean \pm standard deviation. Individual value plots of QCM mass (Figure 4) were created using MINITAB ${ }^{\circledR}$ v.15.1.30 statistical software, $\mathrm{n}=5$. All residual plots for all graphs were checked to ensure the quality of the data obtained. MINITAB ${ }^{\circledR}$ v.15.1.30 was also used to create the regression fitted line plots for deposition analysis using fluorescence and comparisons to QCM deposition (Figure 5); data set was at least $\mathrm{n}=4$ for all dilutions tested. Real-time traces of deposited mass (Figure 3) were made using Microsoft Excel ${ }^{\mathrm{m}}$.

\section{Conclusion}

This study demonstrated the QCM could successfully quantify whole cigarette smoke from two distinct products, generated and diluted using a Borgwaldt RM20S smoke engine and delivered to our whole smoke exposure chamber. The QCM quantified the mass range of delivery of whole smoke particulate from 3R4F reference cigarettes as $25.75 \pm 2.30 \mu \mathrm{g} / \mathrm{cm}^{2}$ to $0.22 \pm 0.03 \mu \mathrm{g} / \mathrm{cm}^{2}$ (within the standard dilution range of 1:5-1:400 (v:v)), and for $1 \mathrm{mg}$ commercial cigarettes as $1.42 \pm 0.26 \mu \mathrm{g} / \mathrm{cm}^{2}$ to $0.13 \pm 0.02 \mu \mathrm{g} / \mathrm{cm}^{2}$ (dilution range 1:5-1:100 (v:v)). When compared to traditional chemical fluorescence method of particulate quantification the QCM measurements were statistically correlated $((\mathrm{p}<0.05)$ with significant regression of $\mathrm{R}^{2}=0.9744$.

The QCM should be easily adapted to assess smoke mass produced from other commercially available smoking machines. Already we have applied the QCM technology to another smoking machine, the Vitrocell $^{\circledR}$ VC10 Smoking Robot, with similar results. Based on the data presented here we have further developed the 'single unit' QCM chamber into a '3-in-1' chamber with 3 identical QCM units installed, one each in the position of a cell culture insert of the BAT whole smoke exposure chamber. This expanded tool has already allowed us to assess potential positional deposition within the exposure chamber and increase replicate number per exposure. Furthermore we are currently testing the QCM module in other commercially available exposure chambers (including the Vitrocell ${ }^{\circledR}$ PT-CF mammalian exposure module) to assess its utility.

In summary, this study outlines the applicability and reliability of the QCM to assess real-time cigarette smoke particle deposition in vitro and suggests the QCM chamber could be a standardised measurement tool to assess and align the particle phase of whole smoke dosimetry in vitro. The exposure chamber alone, although designed to test cigarette whole smoke at ALI, can be used to expose in vitro cultures at the ALI to any aerosol, including environmental pollution, manufactured particles and fibres, aerosolised pharmaceuticals, cosmetics and pesticides or engineered nanoparticles. Therefore the scope of this chamber/QCM combination is vast.

\section{Abbreviations}

QCM: Quartz crystal microbalance; Cmd: Count median diameter; TPM: Total particulate matter; NFDPM: Nicotine free dry particulate matter; HPLC: High performance liquid chromatography; ALl: Air-liquid interface.

\section{Competing interests}

The authors declare that they have no competing interests.

\section{Authors contributions}

JA conceived the study, conducted laboratory work, analysed the data and helped draft the manuscript. SH carried out the QCM work and helped draft the manuscript. DA carried out the spectrofluorometric deposition analysis and helped draft the manuscript. JMCA provided technical expertise and scientific review. MG conceived and reviewed the study and drafted the manuscript. All authors read and approved the final manuscript.

\section{Acknowledgements}

The authors would like to thank Graham Errington at BAT for his guidance on statistical analysis, Tobias Krebs at Vitrocell ${ }^{\circledR}$ Systems and Mark Barber at Borgwaldt-kc for their engineering, mechanical and technical expertise.

Received: 3 May 2012 Accepted: 10 August 2012

Published: 10 September 2012

\section{References}

1. Perfetti TA, Rodgman A: The Complexity of Tobacco and Tobacco Smoke. Beitr. Tabakforsch. Int. 2011, 24:215-232.

2. Adamson J, Azzopardi D, Errington G, Dickens C, McAughey J, Gaça MD: Assessment of an in vitro whole cigarette smoke exposure system: the Borgwaldt RM20S 8-syringe smoking machine. Chem. Cent. J. 2011, 5:50-61.

3. Aufderheide $M$, Knebel JW, Ritter D: An improved in vitro model for testing the pulmonary toxicity of complex mixtures such as cigarette smoke. Exp Toxicol Pathol 2003, 55:51-57.

4. Chalupowicz DG, Frankowski R, Sanchez Y, Kou J, Barnette M, Walsh PT: Cellular responses of primary human bronchial epithelial cells to whole cigarette smoke exposure. Amer.J.of Respiratory and Critical Care Medicine. 2008, 177:199.

5. Fukano Y, Ogura M, Eguchi K, Shibagaki M, Suzuki M: Modified procedure of a direct in vitro exposure system for mammalian cells to whole cigarette smoke. Exp Toxic Pathol. 2004, 55:317-323.

6. Kaur N, Lacasse M, Roy JP, Cabral JL, Adamson JA, Errington G, Waldron KC, Gaca MD, Morin A: Evaluation of precision and accuracy of the Borgwaldt RM20S ${ }^{\circledR}$ smoking machine designed for in vitro exposure. Inhal Toxicol 2010, 22(14):1174-1183.

7. Maunders H, Patwardhan S, Phillips J, Clack A, Richter A: Human bronchial epithelial cell transcriptome: Gene expression changes following acute exposure to whole cigarette smoke in vitro. Am J Physi - Lung Cell Mol Phys. 2007, 292(5):L1248-L1256.

8. Okuwa K, Tanaka M, Fukano Y, Nara H, Nishijima Y, Nishino T: In vitro micronucleus assay for cigarette smoke using a whole smoke exposure system: A comparison of smoking regimens. Exp and Tox Path. 2010, 62:433-440.

9. Phillips J, Kluss B, Richter A, Massey ED: Exposure of bronchial epithelial cells to whole cigarette smoke: assessment of cellular responses. ATLA 2005, 33:239-248.

10. Scian MJ, Oldham MJ, Kane DB, Edmiston JS, McKinney WJ: Characterization of a whole smoke in vitro exposure system (Burghart Mimic Smoker-01). Inhal Toxicol 2009, 21(3):234-243.

11. Thorne D, Wilson J, Kumaravel TS, Massey ED, McEwan M: Measurement of oxidative DNA damage induced by mainstream cigarette smoke in cultured NCI-H292 cells. Mut. Res. 2009, 673(1):3-8. 
12. Teeguarden JG, Hinderliter PM, Orr G, Thrall BD, Pounds JG: Particokinetics in vitro: dosimetry considerations for in vitro nanoparticle toxicity assessments. Toxicol Sci 2007, 95(2):300-312.

13. Paur H-R, Cassee FR, Teeguarden J, Fissan H, Diabate S, Aufderheide M, Kreyling WG, Hänninen O, Kasper G, Riediker M, Rothen-Rutishauser B, Schmid O: In-vitro cell exposure studies for the assessment of nanoparticle toxicity in the lung-A dialog between aerosol science and biology. Journal of Aerosol Science 2011, 42:668-692.

14. Armitage AK, Dixon M, Frost BE, Mariner DC, Sinclair NM: The effect of inhalation volume and breath-hold duration on the retention of nicotine and solanesol in the human respiratory tract and on subsequent plasma nicotine concentrations during cigarette smoking. Beitr Tabakforsch Int. 2004, 21:240-249.

15. Mülhopt S, Diabaté S, Krebs T, Weiss C, Paur HR: Lung toxicity determination by in vitro exposure at the air-liquid interface with an integrated online dose measurement. J Phys Conf Ser 2009, 170:012008.

16. Smith AL, Shirazi HM: Principles of quartz crystal microbalance/heat conduction calorimetry: Measurement of the sorption enthalpy of hydrogen in palladium. Thermochim Acta 2005, 432(2):202-211.

17. Yuwono AS, Lammers PS: Odor pollution in the environment and the detection instrumentation. Agricultural Engineering International: the CIGR Journal of Scientific Research and Development. 2004, 6:1-33.

18. Sauerbrey G: Verwendung von Schwingquarzen zur Wagung dunner Schichten und zur Mikrowagung. Z Phys 1959, 155:206-222.

19. O'Sullivan CK, Guilbault GG: Commercial quartz crystal micro-balances- theory and applications. Biosens Bioelectron 1999, 14(8-9):663-670.

20. Nieuwenhuizen MS, Venema A: Mass-sensitive devices. In Sensors a Comprehensive Survey. Edited by Gopel W, Hesse J, Zemel JN. Germany: VCH, Weinheim; 1999. Vol. 2.

21. Uttenthaler $\mathrm{E}$, Schräml M, Mandel J, Drost S: Ultrasensitive quartz crystal microbalance sensors for detection of M13-Phages in liquids. Biosens Bioelectron 2001, 16(9-12):735-743.

22. Peng B, NingNing L, Wang LM, Zhang WK, Niu ZW, Wang Q, Su ZH: Self-assembly of anisotropic tobacco mosaic virus nanoparticles on gold substrate. SCIENCE CHINA Chem 2011, 54(1):137-143.

23. Kanazawa K, Nam-Joon C: Quartz crystal microbalance as a sensor to characterize macromolecular assembly dynamics. J. Sensors 2009, Article ID 824947, 17 pages

24. Klepeis NE, Ott WK, Switzer P: Real-time measurement of outdoor tobacco smoke particles. J. Air \& Waste Management Association. 2007, 57:522-534.

25. Yeh AC, Turner RS, Jones RK, Muggenburg BA, Lundgren DL, Smith JP: Characterisation of aerosols produced during surgical procedures in hospitals. Aerosol Science and Technology. 1995, 22:151-216.

26. Baron PA, Willeke K: Aerosol measurement: Principles, techniques and applications. 2nd edition.: Wiley Interscience; 2005.

27. Kelly NA, Morgan C: An evaluation of the tapered element oscillating microbalance method for measuring diesel particulate emissions. J Air Waste Manag.Assoc 2002, 52:1362-1377.

28. Carter WL, Hasegawa I: Fixation of tobacco smoke aerosols for size distribution studies. J Colloid Interface Sci 1975, 53:134-141.

29. Allen GA, Sioutas C, Koutrakis P, Reiss R, Lurmann FW, Roberts PT: Evaluation of the TEOM method for measurement of ambient particulate mass in urban areas. JAWMA 1997, 47:682-689.

30. University of Kentucky 2012, 2012. http://www.ca.uky.edu/refcig/3R4F\% 20Preliminary\%20Analysis.pdf.

31. Scian MJ, Oldham M, Miller JH, Kane DB, Edmiston JS, McKinney WJ: Chemical analysis of cigarette smoke particulate generated in the MSB-01 in vitro whole smoke exposure system. Inhal Tox 2009, 21:1040-1052

32. International Organization for Standardisation: Cigarettes - determination of total and nicotine-free dry particulate matter using a routine analytical smoking machine ISO 4387. Geneva, Switzerland: International Standards Organisation; 1991

33. International Organization for Standardisation: Tobacco and tobacco products - Atmosphere for conditioning and testing ISO 3402. Geneva: Switzerland: International Standards Organisation; 1999.
doi:10.1186/1752-153X-6-98

Cite this article as: Adamson et al.: Real-time assessment of cigarette smoke particle deposition in vitro. Chemistry Central Journal 2012 6:98.

\section{Publish with ChemistryCentral and every scientist can read your work free of charge \\ "Open access provides opportunities to our colleagues in other parts of the globe, by allowing anyone to view the content free of charge." W. Jeffery Hurst, The Hershey Company. \\ - available free of charge to the entire scientific community \\ - peer reviewed and published immediately upon acceptance \\ - cited in PubMed and archived on PubMed Central \\ - yours - you keep the copyright \\ Submit your manuscript here: \\ http://www.chemistrycentral.com/manuscript/<smiles>c1ccccc1</smiles> \\ Chemistry Central}

